\title{
Leidenfrost boiling of water droplet
}

\author{
Tadeusz Orzechowski ${ }^{1}$ \\ ${ }^{1}$ Kielce University of Technology, IŚGiE Department, Al. Tysiąclecia PP7, Poland
}

\begin{abstract}
The investigations concerned a large water droplet at the heating surface temperature above the Leidenfrost point. The heating cylinder was the main component of experimental stand on which investigations were performed. The measurement system was placed on the high-sensitivity scales. Data transmission was performed through RS232 interface. The author-designed program, with extended functions to control the system, was applied. The present paper examines the behaviour of a large single drop levitating over a hot surface, unsteady mass of the drop, and heat transfer. In computations, the dependence, available in the literature, for the orthogonal droplet projection on the heating surface as a function of time was employed. It was confirmed that the local value of the heat transfer coefficient is a power function of the area of the droplet surface projection. Also, a linear relationship between the flux of mass evaporated from the droplet and the droplet orthogonal projection was observed.
\end{abstract}

\section{Introduction}

Evaporation of the droplets is studied in many areas of technology [1], [2], [3]. Various investigations conducted into the subject focus on the physics of the phenomenon, which depends strongly on the liquid and its components, thermal and flow conditions, interaction with the surface, etc. Although the problem has been thoroughly analysed, the mechanisms of evaporation are still not fully clear.

Physical processes involved in droplet evaporation are closely related to the surface. At low temperatures, below the saturation temperature, diffusion is a dominant phenomenon in evaporation. The density of the vapour generated at the liquid surface is different from that of the ambient atmosphere. Under the conditions of ideal gas, vapour density is proportional to its molecular mass. If it is water evaporation in the air, rising lighter vapours enhance the convection processes. When the molecular mass of the liquid is greater than $\sim 30 \mathrm{~kg} / \mathrm{kmol}$ (roughly for air), the liquid vapours linger around the surface, high saturation state prevails, and mass exchange is almost entirely diffusive. Such a mechanism was experimentally demonstrated, on the example of water and hexane droplets, in study [4]. At wall superheat of a few degrees, single vapour bubbles can be seen, which initiate nucleate boiling. The droplet lifetime diminishes abruptly, and latent heat of vaporisation allows dissipating large heat fluxes. As regards cooling efficiency, this range is the most advantageous one. Investigations into the dynamics of nucleate boiling in droplets deposited on the rough copper surface and the polished surface with thermal sprayed gold coating were reported in [5]. Heat transfer on the polished surface was found to be considerably lower than on the rough surface. Around the Leidenfrost point, the concentration of vapour inside the droplet is much lower than for pool boiling of water under the same conditions.

Clear differences are observed between nucleate pool boiling and boiling in the droplet. Intense nucleate boiling in the droplet is accompanied by continuous changes in its shape and the area of contact with the substrate. Those phenomena are caused by vapour bubbles that flow inside and depart. The rate and frequency of bubble generation is random in character, which makes evaporation from the droplet unstable and spatially unsteady in this range. Observations of droplets in this range do not reveal structures that are typical for nucleate boiling, namely, depending on the wall superheat, bubble columns, vapour mushrooms and vapour clouds [6], [7]. At wall superheat values below 7 $\mathrm{K}$, vapour bubbles grow relatively slowly and depart not causing significant changes in the droplet shape. Only at the wall superheat above $10 \mathrm{~K}$, bubbles visibly change the droplet circumference. At the bubble growth stage, the resultant force of its buoyancy can be resolved into two components, namely vertical and horizontal ones. The vertical component force causes a change in height, the other - in the boundary of droplet contact with the substrate. The state of the heating surface is decisive for the number of active nucleation centres, which initiate individual bubbles. As the density of nucleation centres on the surface grows with superheat, the nucleate pool boiling becomes more intense. In the limited droplet volume, that becomes a source of instability. When the ratio of the distance between bubbles $S$ to the maximum diameter of the departing bubble $d_{b}$ is in the range $1<$ $\mathrm{S} / \mathrm{d}_{\mathrm{b}}<3$, this instability is substantial. At higher values of this ratio, bubbles do not affect one another. However, when the $\mathrm{S}$ value is lower than unity, bubbles start to coalesce [8], [9]. The maximum heat flux removed from the surface is found at the critical point temperature. Bubbles are formed in densely spaced nucleation centres

Corresponding author: todek@tu.kielce.pl 
in such a way that, at further increase in superheat, bubble columns start coalescing. As a result, the surface is locally dried up, and the density of the removed heat is considerably reduced. Surface temperature grows and the inflowing liquid evaporates abruptly, which makes the whole process unstable. This range is termed a transition boiling regime [10]. The final stage of this regime is the formation of a stable vapour film, which supports the droplet above the heating surface [11], [12]. The first observation of that effect was reported by Johann Gottlob Leidenfrost in A Tract About Some Qualities of Common Water in 1756 and it is named after him to this day. In many technological processes, the element that needs to be cooled has the initial temperature that is higher than Leidenfrost temperature. At the same time, the cooling rate is the parameter that is decisive for the final properties of the top layer and its structure [13], [14]. In technical systems, appropriate control contributes to the optimal conditions of machine operation [15], [16]. To solve the task thus posed, it is necessary to have possibly complete information on the physical processes that occur in droplet evaporation, which despite numerous investigations have not been fully explained.

The aim of this study is to provide the analysis of heat transfer between a large water droplet at atmospheric pressure, and a hot surface at a temperature higher than Leidenfrost temperature.

\section{Experimental Set-up}

Investigations into droplet evaporation at a temperature above the Leidenfrost point were conducted at the stand, the main component of which is a cylindrical copper heater. (see Fig. 1).
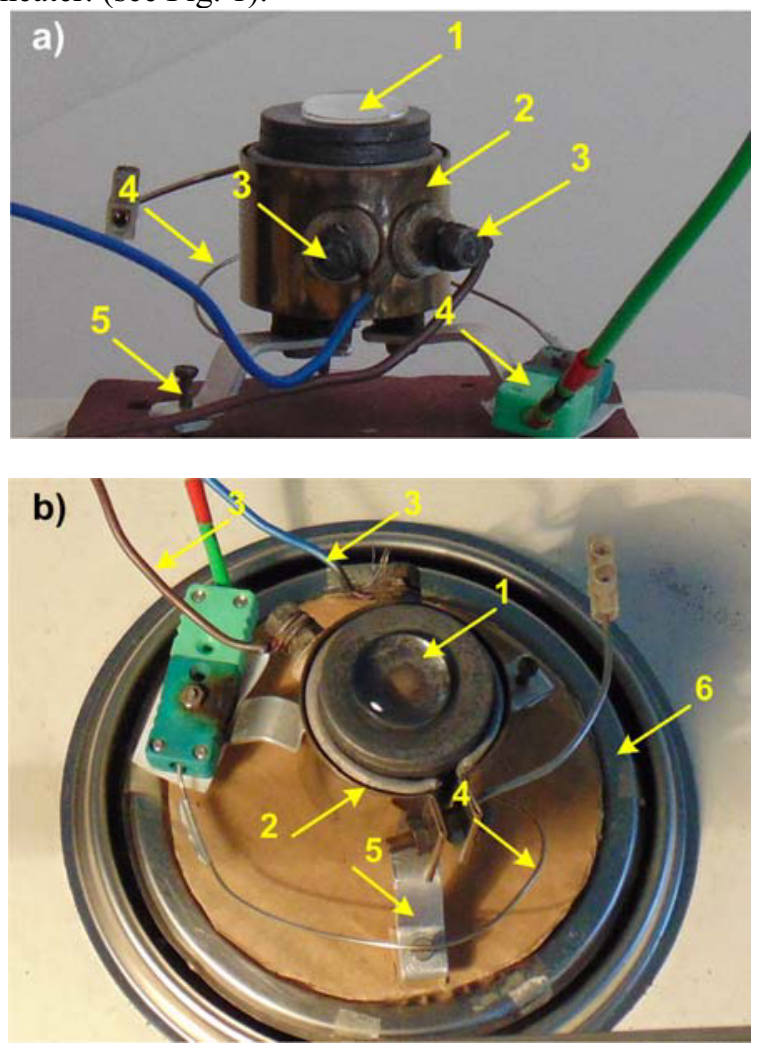

Fig. 1. Photograph of the experimental set-up: a) side view, b) top view 1- droplet, 2 - heater, 3 - electrical power supply, 4 thermocouple, support 5-support pin, 6 - scales-pan.

The cylinder upper surface is shaped like a bowl having a very large radius, which allows centric, stable positioning of the liquid droplet under investigation. Directly under the surface, in the symmetry axis, k-type sheathed thermocouple is placed. It is connected, via suspended conductors, to analogue-digital converter, from which the measured signal is recorded in the computer memory. The heating system consists of a band heater with a resistor placed in the thermal insulation that is capable of continuous operation at the temperatures up to $500{ }^{\circ} \mathrm{C}$. The heater was installed on the cylinder outer surface. The heater is supplied with electric power from the autotransformer by suspended conductors. The output power of the heater is set by voltage, adjusted according to the voltmeter readings. Three support pins were fixed to the heating cylinder. Those support the heating cylinder with the heater, upholding it at approx. $2 \mathrm{~cm}$ above the surface, which prevents excessive heating of the substrate. Additionally, two screws were installed on pin ends. The tightening and loosening of the screws allows precise levelling of the measurement system so that the central position of droplet could be maintained, as shown in Fig.1b.

The complete measurement set was placed on the electronic weighing scales, with the maximum capacity of $500 \mathrm{~g}$. The scales readability is $0.02 \mathrm{~g}$, and sensitivity is $0.001 \mathrm{~g}$. The magneto-electric transducer converts the load to electric signal. The factory setting of the transducer is responsible for precise conversion of timevariable load. Actual readings of the scales are displayed on the liquid crystal panel and the digital signal is available through the RS232 interface, with which the scales is equipped. The interface is used for direct communication with the computer. To that end, the author-designed program was developed to perform the control. The program has, among others, functions for the scales switching on/off, tarring, setting of the sampling frequency and storing the scales readings. The program also makes it possible to temporarily stop the registration and re-start, e.g. when a successive measurement is recorded, after previously tarring the scales, if necessary.

The supply conductors and the compensation one of the thermocouple are suspended from coupled supports that make a rigid and stable construction. The resilience of conductors produces time-variable load, the value of which also depends on temperature. The impact of this phenomenon on the scales readings was effectively eliminated due to several-hour conditioning of the measurement system with electric power supplied. An additional and desirable effect produced in this way was thermal stabilisation. Prior to the start of measurements, a few series of the scales readings were registered to check the stability of readings and repeatability of results. The detailed description of the measurement procedure, together with the analysis of uncertainties were presented in [11] and [17]. 


\section{Results and discussion}

The investigations outcome is a continuous measurement of the mass of evaporating droplet deposited on the upper surface of the heating cylinder (see Fig.1b.), the temperature of which is higher than Leidenfrost temperature. An exemplary set of measurement data is shown in Fig. 2.

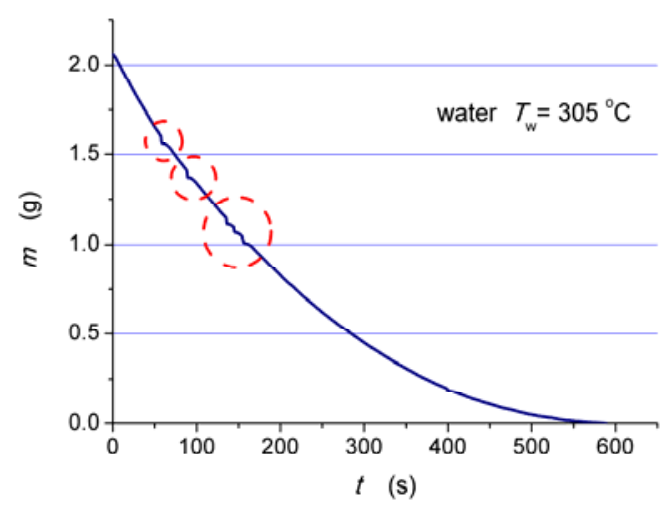

Fig. 2. Evaporation of the water droplet on the wall at the temperature $T_{\mathrm{w}}=305{ }^{\circ} \mathrm{C}$ with marked regions of departing bubbles.

The change in the droplet mass over time is shown for the pre-set temperature of the heating cylinder. The mean value of the latter was $T_{\mathrm{w}}=305{ }^{\circ} \mathrm{C}$. The analysis of the measurement always started after a while, at the instant when the droplet mass was $\sim 2 \mathrm{~g}$. In this way, it was possible to eliminate the period of the initial stabilization of the system, related to the drop deposition. The frequency of the scales readings was always the same and equalled $1 \mathrm{~Hz}$. Because of a growing relative error related to the accuracy of the scales used in the experiment, the measurements ended when the droplet was a few times greater than the scales sensitivity [11].

For large mass droplets, different types of instabilities are observed, which result from complex convective motions inside the droplet [18], [19]. Among others, bubbles appear, which grow and escape. An exemplary instant of a departure of a few bubbles is indicated in Fig. 2. The phenomenon fades away with time as the droplet mass decreases.

Evaporation of the droplet levitating above a hot surface is an extremely complex process, in which the phenomena of heat and mass transfer coincide. However, the greatest portion of heat transfer proceeds between the heating surface and the droplet bottom. If it is assumed that evaporation from the droplet upper surface is small compared with the amount of heat removed from the bottom surface, and overall heat transfer coefficient contains convective and radiative components, i.e. $\alpha=\alpha_{\mathrm{c}}+\alpha_{\mathrm{r}}$, heat balance can be written, which implies change of droplet mass $m$ over time $t$ :

$$
\alpha\left(T_{w}-T_{d}\right) A=-\left(c_{p}\left(T_{s}-T_{d}\right)+h_{f g}\right) \frac{\mathrm{d} m}{\mathrm{~d} t}
$$

where $T_{\mathrm{w}}, T_{\mathrm{d}}$ and $T_{\mathrm{s}}$ are surface, droplet and saturation temperatures, respectively, $A-$ the droplet orthogonal projection onto the heating surface, $c_{\mathrm{p}}$ - specific heat, and $h_{\mathrm{fg}}-$ phase change enthalpy at the saturation temperature, here, at atmospheric pressure.

Using the measured change in the droplet mass over time, on the basis of heat balance (1), it is possible to determine local values of the heat transfer coefficient acc. to the procedure reported in [17]. To this end, by employing smoothing and differentiation procedures available in many computational packages, a change in mass flux over time is determined. The results obtained for the droplet of concern are presented in Fig. 3. Distinct disturbances can also be identified in the figure for the first 170 seconds. They occur for the droplet mass above $\sim 1.3 \mathrm{~g}$. Below that value, the disturbances tend to terminate.

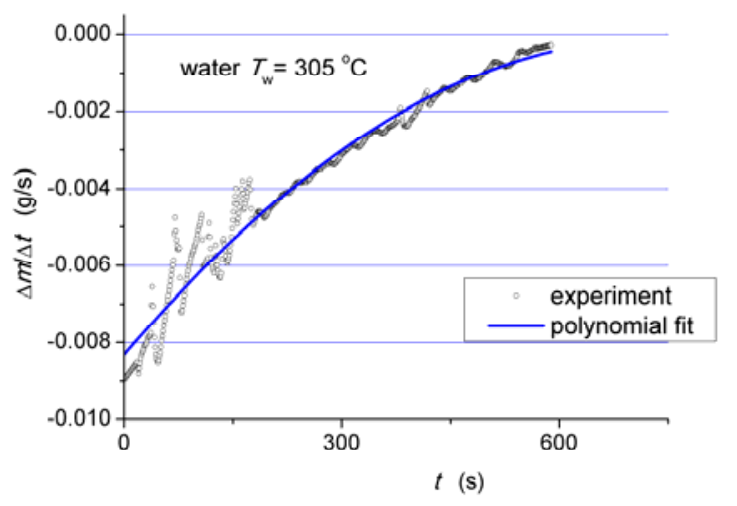

Fig. 3. Changes in the mass flux during evaporation from the droplet.

Different formulas for expressing a change in the droplet orthogonal projection onto the heating surface can be found in literature. Usually, those are timedependent power functions. One of the formulas has the following form [20]:

$$
\frac{A(t)}{A_{0}}=C_{B}\left(a^{t}-C_{A}\right)
$$

where $A_{\mathrm{o}}$ is the area at the initial instant, whereas $\mathrm{C}_{\mathrm{A}}$, $\mathrm{C}_{\mathrm{A}}>0$ and $\mathrm{a}<1$ are constants under pre-set and steady state conditions of the experiment.

Figure 3 shows almost exponential increase, in time, of the flux of the mass evaporated from the droplet. Simultaneously, an exponential decrease in the area of the droplet orthogonal projection onto the heating surface (see Eq. 2) is confirmed by the experiment. As can be seen in Fig. 4, an almost linear relationship holds between those two quantities. The figure also shows the region of departing bubbles, the activity of which in the presented measurement series subsides when the droplet mass is below $1.2 \mathrm{~g}$. 


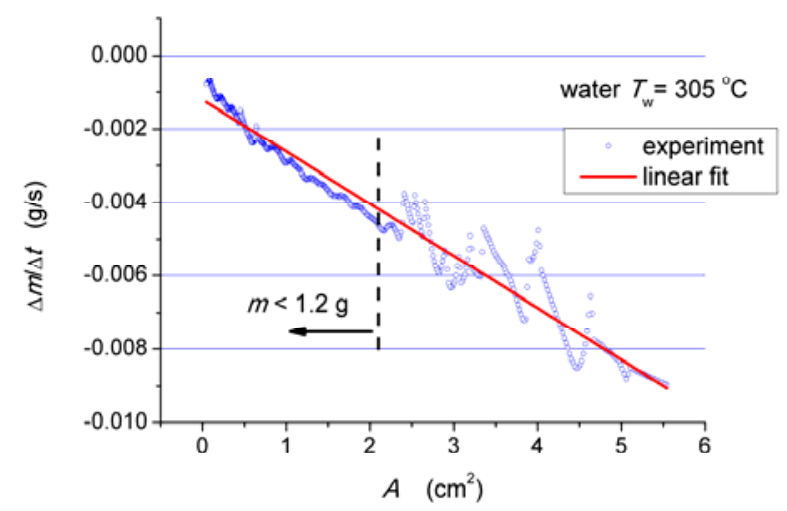

Fig. 4. Mass flux as a function of the droplet orthogonal projection onto the heating surface.

The dependence (2) was used to determine, from equation (1), the local value of the heat transfer coefficient, which as shown in Fig. 5, varies exponentially with the droplet area $A(t)$.

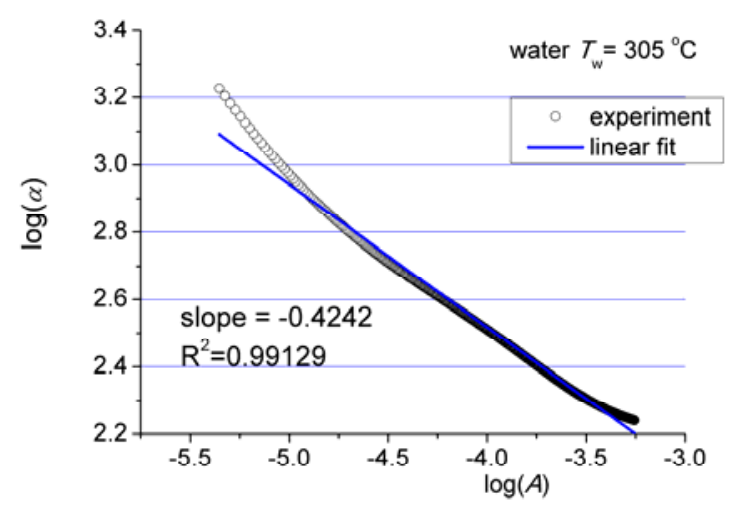

Fig. 5. Local value of the heat transfer coefficient as a function of the area of droplet projection onto the heating surface.

\section{Conclusions}

The investigations concerned a large water droplet at the heating surface temperature above the Leidenfrost point. The heating cylinder was the main component of experimental stand on which investigations were performed. The measurement system was placed on the scales with the sensitivity of $0.001 \mathrm{~g}$ and capacity of up to $500 \mathrm{~g}$ (see Fig. 1). After a few hours of the system conditioning, measurements of the droplet mass in time were taken (see Fig. 2). Data transmission was performed using RS232 interface and the authordesigned software with extended functions for the system control. The analysis of results was carried out employing the droplet heat balance, under the assumption that heat transfer results only from droplet interaction with the heating surface. There, intense evaporation occurs and the remaining components (e.g. evaporation from the droplet upper surface) are negligibly small (see Eq. 1). To make calculations, the dependence, available in the literature, for the droplet orthogonal projection as the function of time was applied (see Eq. 2). It was confirmed that the local value of heat transfer coefficient is a power function of the area of the droplet surface projection (see Fig. 5). The power exponent obtained is approx. -0.42 , which is a slightly lower value than that reported for droplets up to $1 \mathrm{~g}$ in weight [17]. The discrepancy may result from the formula for droplet area dependence on time, that was different from the adopted one. The droplet orthogonal projection was calculated acc. to study [20]. The dependence was given for water droplet deposited over a copper substrate heated to $400{ }^{\circ} \mathrm{C}$, which differs from the quoted studies. As a result, inaccuracies, which are difficult to estimate, may occur in the calculations of temporary values of the heat transfer coefficient (see Fig. 5). Additionally, for large water droplets, above 1g, local generation of bubbles inside droplets is observed. When departing, bubbles disturb the droplet stability (see Figs. 3 and 4). Additionally, a linear relationship between the flux of mass evaporated from the droplet and the droplet orthogonal projection was observed (see Fig. 4).

\section{References}

1. I. Khamis, K.C. Kavvadias, Nucl. Eng. Des. 248, 48 (2012)

2. K.H.M. Abdalrahman, Sabariman, E. Specht, Int. J. Heat Mass Tran. 78, 76 (2014)

3. Y. Wang, N. Zhou, Z. Yang, Y. Jiang, Appl. Therm. Eng. 103, 510 (2016)

4. N. Shahidzadeh-Bonn, S. Rafa, A. Azouni1, D. Bonn, J. Fluid Mech. 549, 307 (2006)

5. S.Ya. Misyura, Int. J. Heat Mass Tran. 71, 197 (2014)

6. S. Nishio, T. Goto, N. Nagaib, Int. J. Heat Mass Tran. 41, 3191 (1998)

7. T.M. Wójcik, EPJ Web of Conferences 25, (2012) 01100

8. M. Shoji, Int. J. Heat Mass Tran. 47, 1105 (2004)

9. V.V. Chekanov, Teplofiz. Vys. Temper. 15, 121 (1977)

10. Y. Mitsutake, S. Illias, K. Tsubaki, M. N. Hasan, M. Monde, Procedia Eng. 105, 5 ( 2015 )

11. T. Orzechowski, S. Wciślik, Energ. Convers. Manage. 76, 918 (2013)

12. T. Orzechowski, S. Wciślik, Exp. Therm. Fluid Sci. 59, 230 (2014)

13. R. Wendelstorf, K.-H. Spitzer, J. Wendelstorf, Int. J. Heat Mass Tran. 51, 4892 (2008)

14. M. Chabičíovský, M. Hnízdil, A.A. Tseng, M. Raudenský, Int. J. Heat Mass Tran. 88, 236 (2015)

15. M.R. Islam, B. Shabani, G. Rosengarten, Appl. Energ. 178, 660 (2016)

16. D. Liu, F.-Y. Zhao, H.-X. Yang, G.-F. Tang, Energy 83, 29 (2015)

17. T. Orzechowski, S. Wciślik, Int. J. Heat Mass Tran. 73, 110 (2014)

18. D. Brutin, B. Sobac, F. Rigollet, C. Le Niliot, Exp. Therm. Fluid Sci. 35, 521 (2011)

19. V.E. Nakoryakov, S.Ya. Misyura, S.L. Elistratov, Int. J. Heat Mass Tran. 55, 6609 (2012)

20. G. Paul, P. K. Das, I. Manna, Exp. Therm. Fluid Sci. 60, 346 (2015) 